\title{
Effects of Pomegranate Seed Oil and Tamoxifen on Mastectomy Breast Cancer Women
}

\author{
${ }^{1,}$ Firas K. Qasim , ${ }^{2,}$ Fikry A. Qadir , ${ }^{3,}$ Karim J. Karim \\ 1,2,3, (Department of Biology, College of Science, Salahaddin University-Erbil)
}

\begin{abstract}
The present study was designed to study the effects of pomegranate and tamoxifen on breast cancer tumor marker (CA 15-3), aromatase enzyme, triglyceride (TG), cholesterol, and lactate dehydrogenase (LDH) in mastectomy women. The results indicates that tumor marker level was increased significantly in women without treatment while the tumor marker was significantly decreased in both pomegranate-tamoxifen combination and tamoxifen group, in addition, the level of aromatase and cholesterol was significantly decreased in pomegranate-tamoxifen combination group when compared with other two remaining groups. LDH level was significantly decreased in both tamoxifen and pomegranate-tamoxifen combination groups when compared with untreated group. However, the level of TG remains unchanged in all groups.
\end{abstract}

Keywords : Pomegranate, tamoxifen, breast cancer, aromatase

\section{INTRODUCTION}

Breast carcinoma is the most common malignancy-affecting women in the US and China, and it is the most common cause of cancer mortality in their population [1]. Approximately 50\% of all women diagnosed with breast carcinoma develop metastatic disease and for these patients, the average survival time from the time of diagnosis of metastatic breast carcinoma ranges from approximately 18-30 months [2].

Tamoxifen is a standard endocrine therapy for the prevention and treatment of steroid hormone receptor-positive breast cancer.Tamoxifen requires enzymatic activation by cytochrome P450 (CYP) enzymes for the formation of active metabolites 4-hydroxytamoxifen and endoxifen. As compared with the parent drug, both metabolites have an approximately 100-fold greater affinity for the estrogen receptor and the ability to inhibit cell proliferation [3].Aromatase is a cytochrome P450 enzyme complex responsible for estrogen biosynthesis and converts androgens, such as testosterone and androstenedione, into the estrogens estradiol and estrone. Aromatase is present in the ovary, placenta, uterus, testis, brain, and extraglandular adipose tissues. Two proteins, cytochrome $\mathrm{P} 450_{\text {arom }}$ and NADPH-cytochrome $\mathrm{P} 450$ reductase, are necessary for enzymatic activity, and the enzyme complex is localized in the smooth endoplasmic reticulum. The aromatase gene, designated CYP19, encodes the cytochrome P450 arom and consists of 10 exons, with the exact size of the gene exceeding 70 kilobases. Aromatase is found in breast tissue, and the importance of intratumoral aromatase and local estrogen production is being unraveled. Effective aromatase inhibitors have been developed as therapeutic agents for estrogen-dependent breast cancer to reduce the growth stimulatory effects of estrogens in breast cancer. Investigations on the development of aromatase inhibitors began in the 1970's and have expanded greatly in the past three decades [3].The polymorphic CYP2D6 is the key enzyme in this biotransformation, and recent mechanistic, pharmacologic, and clinical evidence suggests that genetic variants and drug interaction by CYP2D6 inhibitors influence the plasma concentrations of active tamoxifen metabolites and the outcomes of tamoxifen-treated patients. In particular, nonfunctional (poor metabolizer) and severely impaired (intermediate metabolizer) CYP2D6 alleles are associated with higher recurrence rates [4].

Treatment of hormone-dependent breast cancers has historically focused on manipulation of the level and/or activity of estrogen. Typically, inhibition of estrogen activity is achievable through antagonism of the estrogen receptor (ER) through selective estrogen receptor modulators (SERMs) [5 and 6] or estrogen deprivation via inhibition of estrogen synthesis by the aromatase enzyme [7].Interest in a number of fruits high in polyphenolic compounds has been raised due to their reported chemopreventive potential [8].

\subsection{Design of the study:}

\section{MATERIALS AND METHODS}

Fifty mastectomy women were used in this study, in which they classified into three different groups. First group (control group) consist of 30 mastectomy women, second group (tamoxifen group) consist of 20 mastectomy women and they treated with tamoxifen drug (AstraZeneca-Switzerland) (three tablets "10mg"/day) 
for three months, and third group (pomegranate "LifeExtension-USA"-tamoxifen combination) consist of 20 mastectomy women and they treated with tamoxifen drug (three tablets "10mg"/day) and pomegranate seed oil (three tablets "1g"/day) for three months. The study was carried out during the period of time (May to July 2012)

\subsection{Blood sampling}

Blood samples were taken by $5 \mathrm{cc}$ syringe and put into chilled tubes without ethylene diamine tetra acetic acid (EDTA) and centrifuged at $3000 \mathrm{rpm}$ at $4^{\circ} \mathrm{C}$ for 15 minute; then the sera were stored at $-40 \mathrm{C}^{\circ}$.

\subsection{Estimation of Ca15-3 tumor marker}

Estimation of Ca15-3 tumor marker was done by enzyme-linked immuno sorbent assay (ELISA) technique, which obtained from AccuBind Company (USA).

\subsection{Estimation of Aromatase enzyme}

Estimation of aromatase enzyme was done by enzyme-linked immuno sorbent assay (ELISA) technique, which obtained from Uscn (USA).

\subsection{Estimation of cholesterol, triglyceride and LDH}

The level of cholesterol, triglyceride and LDH were measured by using automated biochemistry analyzer (Kenza 240TX- France).

\subsection{Data Analysis}

Results were expressed as mean \pm standard error of the mean $(M \pm S E M)$. Statistical evaluation of the differences between experimental treatments were determined by performing one-way analysis of variance (ANOVA) in combination with Duncan post hoc analysis, by using statistically available software (SPSS version 11.5). Differences between values were considered statistically significant at $\mathrm{P}$ values $<0.05$.

\section{RESULTS}

A significant decrease of breast tumor marker was observed in pomegranate- tamoxifen combination with mean value $(43.100 \pm 2.307)$ when compared with both tamoxifen alone and breast cancer groups with mean values (55.286 \pm 3.713 and 69.375 \pm 3.520$)$ respectively (Table 1 and Fig. 1).

Table (1): Mean \pm S.E of Effects of Pomegranate + Tamoxifen and Tamoxifen on breast tumor marker

\begin{tabular}{|l|l|}
\hline Groups & CA $15-3 \mathrm{U} / \mathrm{ml}$ \\
\hline Breast cancer & $69.375 \pm 3.520^{\mathrm{c}}$ \\
\hline Pomegranate+ Tamoxifen & $43.100 \pm 2.307^{\mathrm{a}}$ \\
\hline Tamoxifen & $55.286 \pm 3.713^{\mathrm{b}}$ \\
\hline
\end{tabular}

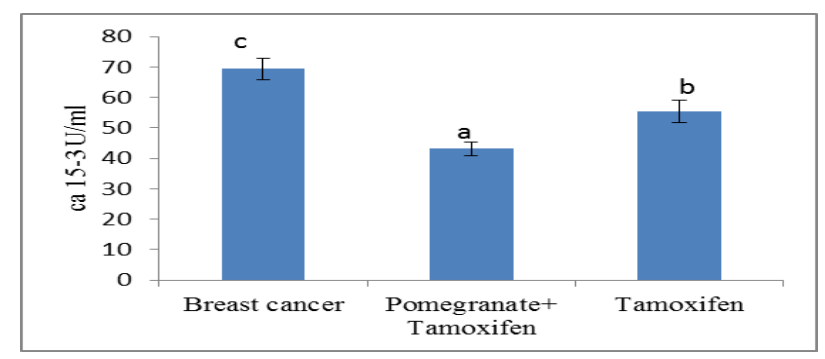

Different letters means significant differences

Similar letters means no significant differences

\section{Figure (1): Effects of Pomegranate + Tamoxifen and Tamoxifen on breast tumor marker}

The level of aromatase enzyme was significantly and clearly decreased in pomegranate and tamoxifen combination with mean value $(4.695 \pm 0.906)$ when compared with breast cancer group with mean value $(8.706$ $\pm 0.821)$, while the level of aromatase in tamoxifen group remains unchanged with mean value $(8.512 \pm 0.737)$ when compared with breast cancer group (Table 2 and Fig. 2). In addition, The level of cholesterol was significantly decreased in pomegranate and tamoxifen combination with mean value $(181.300 \pm 8.164)$ when compared with breast cancer group with mean value $(216.417 \pm 7.033)$, while the level of cholesterol in tamoxifen group remains unchanged with mean value $(212.500 \pm 6.350)$ when compared with breast cancer group (Table 3 and Fig. 3). 
Table (2): Mean \pm S.E of Effects of Pomegranate + Tamoxifen and Tamoxifen on aromatase level

\begin{tabular}{|l|l|}
\hline Groups & Aromatase \\
\hline Breast cancer & $8.706 \pm 0.821^{\mathrm{b}}$ \\
\hline Pomegranate+ Tamoxifen & $4.695 \pm 0.906^{\mathrm{a}}$ \\
\hline Tamoxifen & $8.512 \pm 0.737^{\mathrm{b}}$ \\
\hline
\end{tabular}

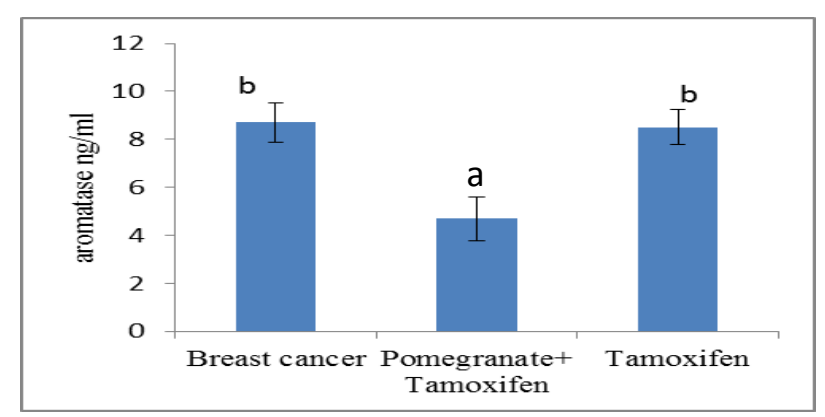

Different letters means significant differences

Similar letters means no significant differences

Figure (2): Effects of Effects of Pomegranate + Tamoxifen and Tamoxifen on aromatase

Table (3): Mean \pm S.E of Effects of Pomegranate + Tamoxifen and Tamoxifen on cholesterol, TG, and LDH levels

\begin{tabular}{|l|l|c|c|}
\hline \multicolumn{1}{|c|}{ Groups } & Cholesterol mg/dl & TG mg/dl & LDH IU/ml \\
\hline Breast cancer & $216.417 \pm 7.033^{\mathrm{b}}$ & $251.583 \pm 13.339^{\mathrm{a}}$ & $951.083 \pm 67.516^{\mathrm{b}}$ \\
\hline Pomegranate+ Tamoxifen & $181.300 \pm 8.164^{\mathrm{a}}$ & $232.400 \pm 22.504^{\mathrm{a}}$ & $506.4 \pm 62.256^{\mathrm{a}}$ \\
\hline Tamoxifen & $212.500 \pm 6.350^{\mathrm{b}}$ & $237.286 \pm 13.506^{\mathrm{a}}$ & $705.5 \pm 58.011^{\mathrm{a}}$ \\
\hline
\end{tabular}

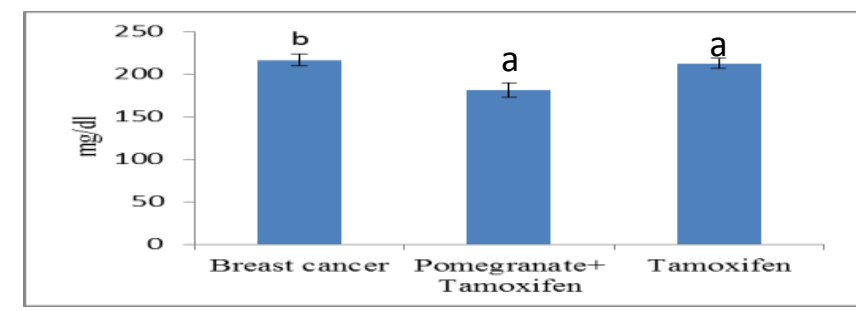

Different letters means significant differences

Similar letters means no significant differences

Figure (3): Effects of Effects of Pomegranate + Tamoxifen and Tamoxifen on cholesterol

The level of TG in both pomegranate and tamoxifen group and tamoxifen group does not show significant change with mean value $(232.400 \pm 22.504$ and $237.286 \pm 13.506)$ respectively when compared with the breast cancer group with mean value (251.583 \pm 13.339$)$ (Table 3 and Fig. 4).

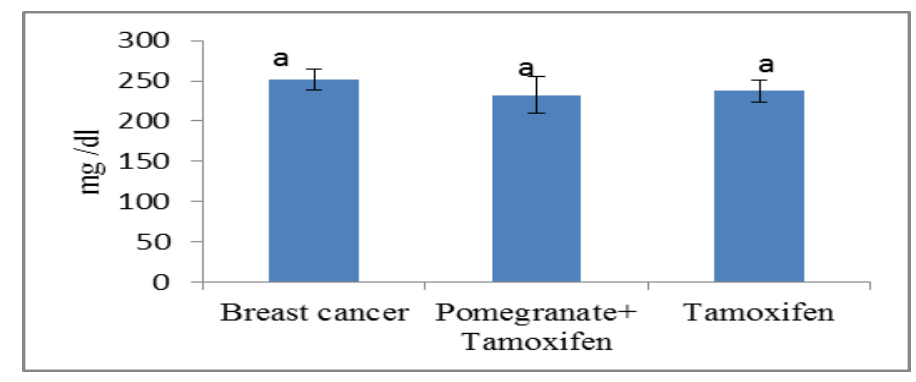

Different letters means significant differences

Similar letters means no significant differences

Figure (4): Effects of Effects of Pomegranate + Tamoxifen and Tamoxifen on triglyceride 
In addition, the level of LDH in both pomegranate and tamoxifen group and tamoxifen group was significantly decreased with mean value $(506.4 \pm 62.256$ and $705.5 \pm 58.011)$ respectively when compared with the breast cancer group with mean value $(951.083 \pm 67.516)$ (Table 3 and Fig. 5).

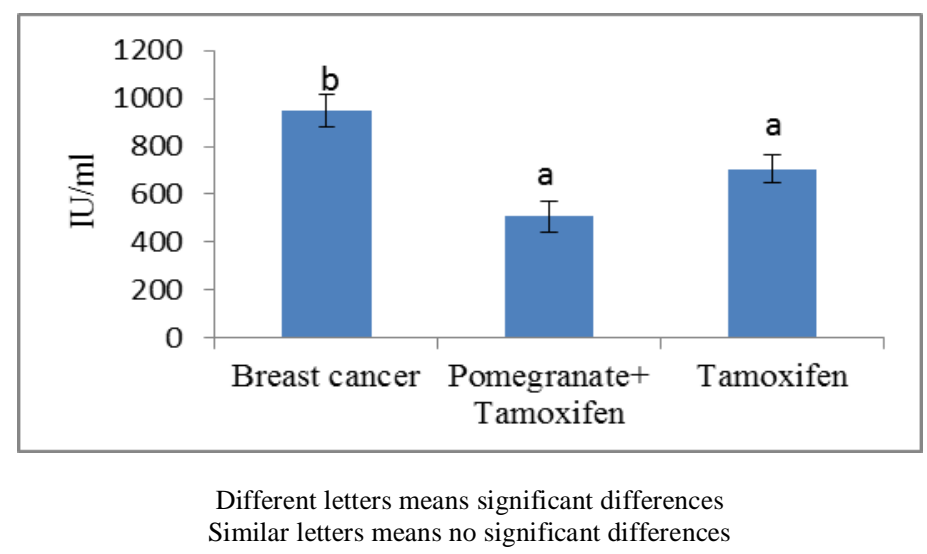

Figure (5): Effects of Effects of Pomegranate + Tamoxifen and Tamoxifen on LDH

\section{DISCUSSION}

The result showed that CA15-3 decreased significantly in pomegranate- tamoxifen combination when compared with both tamoxifen alone and breast cancer groups. Porika et al., (2010) [9] reported that the mean serum levels of CA 15.3 dropped slightly 9 days after mastectomy, however, 27 days later the values were significantly increased among Southern Indian breast cancer patients. Tamoxifen pharmacogenetics focuses on enzymes transforming the prodrug tamoxifen to 30 to 100 times more active metabolites (4-hydroxy tamoxifen and endoxifen). This creates the possibility to identify pharmacogenetic markers that can predict efficacy and may be used in clinical practice [10].

Tamoxifen therapy for breast cancer has become more generally used and it remains a challenge to predict which patients are at greatest risk of relapse and thus, may benefit most from tamoxifen therapy. In these cases, measurement of serum tumors markers may be helpful in detecting the metastatic process, in the subclinical phase itself and assess the response to the treatment in breast cancer patients. Several studies have shown that, in approximately $90 \%$ of the metastatic patients, serum levels of CA 15-3 correlate directly with the response of the disease to the treatment. Hence, elevations of tumor marker levels during the treatment phase could be a promising indicative of disease progression or regression [11].

The antitumor activity of tamoxifen is due to its antiestrogenic activity, mediated by competitive inhibition of estrogen binding to estrogen receptors of the target tissue and blocking the action of biologically active estrogen and estradiol. Consequently, tamoxifen inhibits the expression of estrogen-regulated genes, including growth factors and angiogenic factors secreted by the tumor that may stimulate growth by autocrine or paracrine mechanisms [11].

In addition, the antiproliferative effects of tamoxifen may relate to its inhibition of protein kinase $\mathrm{C}$ and it's binding to calmodulin, a protein that plays a role in DNA synthesis [11].

Tamoxifen blocks estrogen binding to the estrogen receptor (ER), making patients with ER-positive disease more likely to respond than those with ER-negative disease, tamoxifen prevents mammary cancer in rats so the drug could reduce the incidence of primary breast cancer, and long-term treatment was better than shortterm treatment to prevent rat mammary carcinogenesis; therefore, longer adjuvant therapy with tamoxifen should be superior to short-term adjuvant therapy [12].

The anti-estrogen tamoxifen was the first of a class of drugs, now referred to as selective estrogen receptor modulators, to be used clinically. Originally developed as an oral contraceptive, tamoxifen was actually found to induce ovulation in subfertile women and was marketed for this indication instead. In the early 1970s, following promising preliminary results in post-menopausal breast cancer patients [13], tamoxifen was reinvented as an anticancer drug and is now the most widely used adjuvant therapy in the treatment of breast cancer. Randomized clinical trials have demonstrated that adjuvant tamoxifen results in increased disease-free survival and a decrease in breast cancer recurrence rates saving thousands of lives each year [14]. 
Pomegranate juice (Punica granatum L) has been shown to be high in antioxidant activity, which is generally attributed to its high polyphenol content. Pomegranate juice is produced by squeezing of the whole fruit, leading to a high content of polyphenols, among which ellagitannins (ETs) predominate [8]. Pomegranate juice and its purified ETs have been shown to inhibit cancer cell proliferation [15] and inflammatory cell signaling [16] in vitro. Previous work has shown that ETs from pomegranate juice are hydrolyzed to ellagic acid (EA) which is then taken up intact and metabolized by human colonic microflora to 3,8-dihydroxy-6Hdibenzo[ b,d]pyran-6-one derivatives (urolithin A and B) [17, 18 and 19]. These compounds have been shown to inhibit the growth of human prostate and breast cancer cell lines [20]. In vitro studies have demonstrated that urolithin A (UA) and urolithin B (UB) are taken up intact in MCF-7 breast cancer cells and converted to sulfate and glucuronate metabolites. In addition, it has been shown that UB is partially converted to UA in MCF-7 cells [21]. Finally, in vivo studies have shown that pomegranate extract and UA both inhibited the growth of LAPC-4 derived tumors in SCID mice [20].

One of the most well-established signaling pathways mediating inflammatory responses relevant to cancer is the nuclear factor $-\kappa B(N F-\kappa B)$ pathway. NF- $\kappa B$ represents a family of transcription factors that modulate expression of genes with diverse functions. The activity of NF- $\kappa \mathrm{B}$ is regulated by the inhibitor of $\kappa \mathrm{B}$

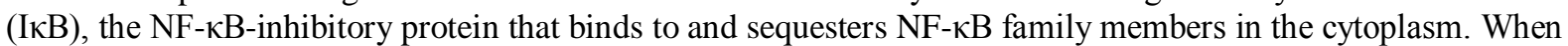
the $N F-\kappa B$ pathway is activated, I $\mathrm{KB}$ is phosphorylated by I $\kappa \mathrm{B}$ kinase, which phosphorylates I $\mathrm{B}$ at serine residues 32 and 36 [22]. Phosphorylated $\mathrm{I} \kappa \mathrm{B}$ is subjected to ubiquitination and proteasome-mediated degradation, which results in the translocation of $\mathrm{NF}-\kappa \mathrm{B}$ to the nucleus, where it functions as a transcription factor.

Interestingly, pomegranate extract (PE) has been shown to inhibit NF- $\kappa$ B in normal human cells, [23]. The result showed that level of aromatase significantly decreased in pomegranate-tamoxifen combination when compared with tamoxifen alone and breast cancer group, and there is no any significant change in level of aromatase in breast cancer group and tamoxifen alone.As we mentioned in the introduction that aromatase is a cytochrome P450 enzyme complex responsible for estrogen biosynthesis and converts androgens, such as testosterone and androstenedione, into the estrogens estradiol and estrone. Because estrogen has an important role in breast cancer production, so aromatase level increases in breast cancer.So if any mutation occurs in aromatase gene, the level of breast cancer decreases. Study of 204 Japanese women has shown that the single amino acid substitution in aromatase of tryptophan by arginine at codon 39 was significantly associated with a decrease in breast cancer risk [24]. Those authors speculated that this polymorphism may reduce the activity of the CYP19 enzyme, consequently resulting in a lower production of estrogens.

Two primary approaches have been developed to reduce the growth-stimulatory effects of estrogens in breast cancer: 1) interfering with the ability of estrogen to bind to its receptor; and 2) decreasing circulating levels of estrogen. Antiestrogens compete for binding to the estrogen receptors and reduce the number of receptors available for binding to endogenous estrogen. This approach has proven effective as an anticancer strategy $[25,26]$ and has led to the development of efficacious antiestrogens, such as the drug tamoxifen, that exhibit minimal toxicity. Inhibition of aromatase is the second approach for reducing growth-stimulatory effects of estrogens. Effective aromatase inhibitors have been developed as therapeutic agents for controlling estrogendependent breast cancer. Investigations on the development of aromatase inhibitors began in the 1970s and have expanded greatly in the past three decades. Research summaries of aromatase inhibitors have been presented at international aromatase conferences [27, 28]. Inhibition of testosterone-induced cell proliferation was observed with pomegranate treatment as would be expected from the aromatase assay results. This suggests that the inhibition of aromatase, and therefore estrogen production in the cell, is one mechanism through which pomegranate may inhibit breast cancer cell proliferation [8].

The result showed that the level of cholesterol significantly decreased in pomegranate-tamoxifen combination when compared with other groups, and surprisingly the level of cholesterol didn't change in tamoxifen group when compared with breast cancer group.Studies suggest that cholesterol and cancer are closely associated, where cholesterol tends to accumulate in the cells comprising solid tumors [29, 30, and 31]. The mechanisms of cholesterol homeostasis are often dysregulated in tumors so that cholesterol deposition is favored [32]. These findings are particularly interesting in the context of developed countries such as the United States where diets that are high in cholesterol and fatty acids are thought to be associated with a higher incidence of breast cancer [32, 33]. 
In normal nonmalignant tissues intracellular cholesterol is closely monitored and adjusted to maintain appropriate cholesterol levels [34]. The accumulation of cholesterol that is often seen in breast cancer is most likely due to alterations in cholesterol acquisition, efflux and/or transport within the breast cancer cells [35], also Liu et al.,(2012) [36] reported that cholesterol level increased in breast cancer women.In our study the level of cholesterol not altered in tamoxifen group, and we can explain this result which may be, in part, due to inherited genetic differences in the cellular receptors [37]. Tamoxifen acts via binding and modulation of the ERs, which are members of the nuclear steroid receptor superfamily.

Two subsets of human ERs have been identified: ER- $\alpha$ and ER- $\beta$. These receptors are products of different genes, designated ESRI and ESR2, respectively [38]. The expression of these receptors is influenced by genetic polymorphisms, as is the case for other members of the nuclear receptor superfamily [37]. Singlenucleotide polymorphisms (SNPs) in the genes coding for the ERs may therefore explain some of the variability seen in the response to tamoxifen, including changes in lipid profile. The interaction of the ERs with each other may also be important, as studies have suggested that ER- $\beta$ modulates some of the responses resulting from activation of ER- $\alpha$ target [39]. Other studies suggest that tamoxifen decrease the level of cholesterol [40, 41] Pomegranate juice consumption decreased LDL susceptibility to aggregation and retention and increased the activity of serum paraoxonase (an HDL-associated esterase that can protect against lipid peroxidation) by $20 \%$ [42].

Pomegranate flowers and fruit extracts exhibited high activity on lowering circulation lipid and modifying heart disease risk factors in diabetic animals and humans with hyperlipidemia [43], but the mechanism is still unknown. It has been found that the pomegranate leaf extract (PLE), containing abundant tannins, had a strong lipid-lowering action, that is, decreased the plasma levels of total cholesterol in hyperlipemic animals after a long-term of oral administration [44]. The level of triglyceride decreased in pomegranate-tamoxifen combination and tamoxifen alone when compared with breast cancer group but this decreasing is not significant. Same result was reported by Gupta et al., (2006) [40] when he studied the effects of tamoxifen therapy on plasma lipid profile in patients of breast cancer.

Huang et al., (2005) [43] reported that oral administration of pomegranate leaf extract improves excess cardiac triglyceride accumulation, accompanied by improvement of hypertriglyceridemia and hypercholesterolemia in rats. The level of LDH significantly decreased in pomegranate-tamoxifen combination and tamoxifen alone when compared with breast cancer group. The accumulation of lactate in tumor cells was first described by Warburg and is associated with aerobic glycolysis [45]. Clinical studies showed that high lactate levels are associated with the subsequent development of metastases [46]. In cancer patients, serum total $\mathrm{LDH}$ levels are often increased, and the gene for $L D H-A$ protein is often upregulated in tumors [47].

Glucose and glutamine are the major carbon sources for rapidly proliferating tumors, providing precursors for nucleic acids, proteins, and lipids, as well as metabolic-reducing capability (NADPH). Pyruvate is largely derived from both glucose and glutamine metabolism; it can be converted to lactate by the LDH complex and/or enter the TCA cycle for conversion to $\mathrm{CO}_{2}$ and ATP. The conversion of pyruvate to lactate is also catalyzed by $\mathrm{LDH}$ which is a reversible reaction that results in the formation of $\mathrm{NAD}^{+}$, which is necessary for further glycolysis. LDH isoenzymes are found in almost all eukaryotic cells and tissues, reflecting the importance of this metabolic step. As a consequence of increased glucose and glutamine metabolism, tumors secrete lactate, alanine, and $\mathrm{NH}^{+}{ }^{4}$. When oxygen is available, the accumulated and exogenous lactate can be reutilized and converted back to pyruvate, where it is further oxidized to $\mathrm{CO}_{2}$ and $\mathrm{H}_{2} \mathrm{O}$ in the TCA cycle, generating ATP and $\mathrm{NAD}^{+}$[48]. It was shown that $\mathrm{LDH}-\mathrm{A}$ is required for the maintenance and progression of many tumors [47, 49, and 50], but the mechanisms by which LDH-A facilitates tumor progression are poorly understood [51]. The significant increase in serum LDH is associated with increased lipid peroxidation which reflecting hepatic membrane damage [52]. Pomegranate rind aqueous extract exhibit strong antioxidant activity [53], scavenging free radicals and ameliorate the damage and decrease the level of LDH. This result is in agreement with other studies which was done and causes decrease in the level of LDH like [54, 55].

\section{CONCLUSION:}

The study suggests that supplementation of pomegranate along with tamoxifen to breast cancer patients reduces the serum tumor marker levels of CA 15-3, Aromatase and LDH thereby offering better cancer prognosis by reducing the risk of developing cancer recurrence and metastasis. 


\section{REFERENCES}

[1] EM John, JL Hopper, JC Beck, JA Knight, SL Neuhausen, RT Senie, A Ziogas, LL Andrulis, H Anton-Culver, N Boyd, SS Buys, MB Daly, FP O’Malley, RM Santella, MC Southey, VL Venne, DJ Venter, DW West, AS Whittemore, and D Seminara. The Breast Cancer Family Registry, an infrastructure for cooperative multinational, interdisciplinary and translational studies of the genetic epidemiology of breast cancer. Breast Cancer Res 6, 2004, 375-389

[2] X Li, Z Meng, W Guo and J Li. Treatment for liver metastases from breast cancer, Results and prognostic factors. World $J$ Gastroenterol 11(24), 2005,3782-3787

[3] GE Timm. Final Detailed Review Paper On Aromatase. Epa Contract Number 68-W-01-023 Work Assignments 2-7 and 5-5, TASK 2, 2005

[4] H Brauch, T E Mürdter, M Eichelbaum and M Schwab. Pharmacogenomics of Tamoxifen Therapy. Clinical Chemistry 55(10), 2009, 1770-1782

[5] B Fisher, J Dignam, and N Wolmark. Tamoxifen in treatment of intraductal breast cancer, National Surgical Adjuvant Breast and Bowel Project B-24 randomised controlled trial. Lancet 353(9169), 1999, 1993-2000

[6] B Fisher, JP Costantino, DL Wickerham. Tamoxifen for the prevention of breast cancer, current status of the National Surgical Adjuvant Breast and Bowel Project P-1 study. J Natl Cancer Inst 97(22), 2005,1652-62

[7] F Boccardo, A Rubagotti, D Aldrighetti. Switching to an aromatase inhibitor provides mortality benefit in early breast carcinoma, pooled analysis of 2 consecutive trials. Cancer 109(6), 2007, 1060-7

[8] L S Adams, Y Zhang, N P Seeram, D Heber and S Chen. Pomegranate Ellagitannin-Derived Compounds Exhibit Antiproliferative and Anti-aromatase Activity in Breast Cancer Cells In Vitro. Cancer Prev Res (Phila). 3(1), 2010, 108-113

[9] M Porika, N Malotu, U K Veldandi, Yadala and S Abbagani...Evaluation of Tumor Markers in Southern Indian Breast Cancer Patients. Asian Pacific J Cancer Prev, 11, 2010,157-159

[10] V O Dezentjé, H Guchelaar, J WR Nortier. CJH van deVelde and H Gelderblom. Clinical Implications of CYP2D6 Genotyping in Tamoxifen Treatment for Breast Cancer. Clin Cancer Res 15, 2009, 15-21

[11] V G Premkumar, S Yuvaraj, K Vijayasarathy, S G D Gangadaran and P Sachdanandam. Effect of Coenzyme Q10, Riboflavin and Niacin on Serum CEA and CA 15-3 Levels in Breast Cancer Patients Undergoing Tamoxifen Therapy. Biol. Pharm. Bull. 30(2), 2007, 367-370

[12] VC Jordan and M Morrow. Tamoxifen, Raloxifene, and the Prevention of Breast Cancer. Endocrine Rev 20(3), 1999, 253-278

[13] M P Cole, C T A Jones and I D H Todd. New antioestrogenic agent in late breast cancer-early clinical appraisal of ICI46474. Br. J. Cancer 25, 1971, 270-275

[14] I N H White. The tamoxifen dilemma. Carcinogenesis, 20, 1999, 1153-1160

[15] NP Seeram, LS Adams and SM Henning. In vitro antiproliferative, apoptotic and antioxidant activities of punicalagin, ellagic acid and a total pomegranate tannin extract are enhanced in combination withother polyphenols as found in pomegranate juice. $J$ Nutr Biochem 16(6), 2005,360-7

[16] LS Adams, NP Seeram and BB Aggarwal. Pomegranate juice, total pomegranate ellagitannins, and punicalagin suppress inflammatory cell signaling in colon cancer cells. J Agric Food Chem 54(3), 2006, 980-985

[17] NP Seeram, SM Henning and Y Zhang. Pomegranate juice ellagitannin metabolites are present in human plasma and some persist in urine for up to 48 hours. $J$ Nutr 136(10), 2006, 2481-5

[18] B Cerda, C Soto and MD Albaladejo. Pomegranate juice supplementation in chronic obstructive pulmonary disease, a 5-week randomized, double-blind, placebo-controlled trial. Eur J Clin Nutr 60(2), 2006, 245-53

[19] SU Mertens-Talcott, P Jilma-Stohlawetz, J Rios, L Hingorani and H Derendorf. Absorption, metabolism, and antioxidant effects of pomegranate (Punica granatum 1.) polyphenols after ingestion of a standardized extract in healthy human volunteers. $J$ Agric Food Chem 54(23), 2006, 8956-61

[20] NP Seeram, WJ Aronson and Y Zhang. Pomegranate ellagitannin-derived metabolites inhibit prostate cancer growth and localize to the mouse prostate gland. J Agric Food Chem 55(19), 2007, 7732-7

[21] M Larrosa, A Gonzalez-Sarrias, MT Garcia-Conesa, FA Tomas-Barberan and JC Espin. Urolithins, ellagic acid-derived metabolites produced by human colonic microflora, exhibit estrogenic and antiestrogenic activities. J Agric Food Chem 54(5), 2006, 1611-20

[22] AS Baldwin. Series introduction, the transcription factor NF- $\kappa$ B and human disease. J Clin Invest 107, 2001, 3-6.

[23] S Ahmed, N Wang, BB Hafeez, VK Cheruvu and TM Haqqi. Punica granatum L. extract inhibits IL-1 $\beta$-induced expression of matrix metal-loproteinases by inhibiting the activation of MAP kinases and NF- $\kappa$ B in human chondrocytes in vitro. $J$ Nutr $\mathbf{1 3 5}$, 2005, 2096-102.

[24] Y Miyoshi, K Iwao, N Ikeda, C Egawa and S Noguchi. Breast cancer risk associated with polymorphism in CYP19 in Japanese women. Int J Cancer 89, 2000, 325-328

[25] VC Jordan. Tamoxifen, toxicities and drug resistance during the treatment and prevention of breast cancer. Annu Rev Pharmacol Toxicol 35, 1995, 195-211

[26] Carmichael PL. 1998. Mechanisms of action of antiestrogens, relevance to clinical benefits and risks. Cancer Invest 16,604-611

[27] ER Simpson, A Bhatnagar, AM Brodie, RW Brueggemeier, S Chen, R DeCoster, M Dowsett, N Harada, F Naftolin, Y Osawa, P Plourde, MJ Reed, RJ Santen. Proceedings of the Fourth International Aromatase Conference. J Steroid Biochem Mol Biol 61, $1997,107-425$

[28] ER Simpson. Aromatase 2000, Sixth International Aromatase Conference. J Steroid Biochem Mol Biol 79, 2001, 1-314

[29] E A Mady. Association between estradiol, estrogen receptors, total lipids, triglycerides, and cholesterol in patients with be nign and malignant breast tumors. J Steroid Biochem and Molec Biol 75(4-5), 2000, 323-328

[30] M R Freeman and K R Solomon. Cholesterol and prostate cancer. J Cellu Biochem 91(1), 2004, 54-69

[31] Y C Li, M J Park, S K Ye, CW Kim and Y N Kim. Elevated levels of cholesterol-rich lipid rafts in cancer cells are correlated with apoptosis sensitivity induced by cholesteroldepleting agents. The Am J Path 168(4), 2006, 1107-1118

[32] G Llaverias, C Danilo and I Mercier. Role of cholesterol in the development and progression of breast cancer. The Am J Path 178(1), 2011, 402-412

[33] S S Coughlin and D U Ekwueme. Breast cancer as a global health concern. Cancer Epidem 33(5), 2009, $315-318$

[34] P G Yancey, A E Bortnick, G Kellner-Weibel, M de la Llera-Moya, M C Phillips and G H Rothblat. Importance of different pathways of cellular cholesterol efflux. Arterioscl Thromb and Vasc Biol 23(5), 2003, 712-719

[35] B J Martin and K L van Golen. A Comparison of Cholesterol Uptake and Storage in Inflammatory and Noninflammatory Breast Cancer Cells. Int J Breast Cancer 2012,1-10 
[36] YL Liu, HX Qian, L Qin, XJ Zhou, B Zhang and X Chen. Association of serum lipid profile with distant metastasis in breast cancer patients. Zhonghua Zhong Liu Za Zhi. 34(2), 2012, 129-31

[37] F Massart. Human races and pharmacogenomics of effective bone treatments. Gynecol Endocrinol 20, 2005, 36-44

[38] GG Kuiper, E Enmark, M Pelto-Huikko, S Nilsson and JA Gustafsson. Cloning of a novel receptor expressed in rat prostate and ovary. Proc Natl Acad Sci 93, 1996, 5925-5930

[39] JM Hall and DP McDonnell. The estrogen receptor beta-isoform (ERbeta) of the human estrogen receptor modulates ERalpha transcriptional activity and is a key regulator of the cellular response to estrogens and antiestrogens. Endocrinology 140, 1999, 5566-5578.

[40] S Gupta, VR Tandon, B Kapoor, A Gupta, GD Gupta and V Khajuria. Effects of tamoxifen therapy on plasma lipid profile in patients of breast cancer. J Assoc Physicians India 54, 2006, 183-6.

[41] NI Ntukidem, AT Nguyen, V Stearns, M Rehman, A Schott, T Skaar, Y Jin, P Blanche, L Li, S Lemler, J Hayden, RM Krauss, Z Desta, DA Flockhart and DF Hayes. Estrogen Receptor Genotypes, Menopausal Status, and the Lipid Effects of Tamoxifen. Clin Pharmacol Ther. 83(5), 2008, 702-710

[42] M Aviram, L Dornfeld, M Rosenblat, N Volkova, M Kaplan, R Coleman, T Hayek, D Presser and B Fuhrman. Pomegranate juice consumption reduces oxidative stress, atherogenic modifications to LDL, and platelet aggregation, studies in humans and in atherosclerotic apolipoprotein E-deficient mice. Am J Clin Nutr 71, 2007, 1062-76

[43] T H Huang, G Peng, B P Kota, G Q Li, J Yamahara, B D Roufogalis and Y Li. Pomegranate flower improves cardiac lipid metabolism in a diabetic rat model, role of lowering circulating lipids. Br J Pharma 145, 2005, 767-774

[44] F Lei, XN Zhang, W Wang, DM Xing, WD Xie, H Su and LJ Du. Evidence of anti-obesity effects of the pomegranate leaf extract in high-fat diet induced obese mice. Int J Obes 31, 2007, 1023-1029

[45] O Warburg. On respiratory impairment in cancer cells. Science 1956,124,269-70. Cited from I Serganova , A Rizwan , X Ni , S B Thakur , J Vider , J Russell , R Blasberg and J A Koutcher. Metabolic Imaging, A Link between Lactate Dehydrogenase A, Lactate, and Tumor Phenotype. Clin Cancer Res 1(17), 2011, 6250

[46] S Walenta and WF Mueller-Klieser. Lactate, mirror and motor of tumor malignancy. Semin Radiat Oncol 14, $2004,267-74$.

[47] VR Fantin, J St-Pierre and P Leder. Attenuation of LDH-A expression uncovers a link between glycolysis, mitochondrial physiology, and tumor maintenance. Cancer Cell 9, 2006, 425-34

[48] C Granchi, S Bertini, M Macchia and F Minutolo. Inhibitors of lactate dehydrogenase isoforms and their therapeutic potentials. Curr Med Chem 17, 2010, 672-97.

[49] A Le, CR Cooper, AM Gouw, R Dinavahi, A Maitra, and LM Deck. Inhibition of lactate dehydrogenase A induces oxidative stress and inhibits tumor progression. Proc Natl Acad Sci USA 107, 2010, 2037-42

[50] P Seth, A Grant, J Tang, E Vinogradov, X Wang and R Lenkinski. On-target inhibition of tumor fermentative glycolysis as visualized by hyperpolarized pyruvate. Neoplasia 13, 2011, 60-71

[51] I Serganova, A Rizwan, X Ni, S B Thakur, J Vider, J Russell, R Blasberg and J A Koutcher. Metabolic Imaging, A Link between Lactate Dehydrogenase A, Lactate, and Tumor Phenotype. Clin Cancer Res 1(17), 2011, 6250

[52] P Morliere, A Moysan, R Santus, JC Huuppe and L Ubertret. UVA induced lipid peroxidation in cultured human fibroblasts Biochem. Biophys. Acta 1084, 1991, 261

[53] M K Chidambara, GK Jayaprakasha and RP Singh. Studies on antioxidant activity of pomegranate (Punica granatum) peel extract using in vivo models .J Agric Food Chem 50, 2002, 4791

[54] E A M Khalil. A hepatoprotective effect of an aqueous extract of pomegranate (Punica granatum L.) rind against acetaminop hen treated rats. The Egyptian Journal of Hospital Medicine 16, 2004, 112-118

[55] M Mohan, P Patankar, P Ghadi and S Kasture. Cardioprotective potential of Punica granatum extract in isoproterenol-induced myocardial infarction in Wistar rats. J Pharmacol Pharmacother 1(1), 2010, 32-37 